\title{
Dieta de Phytotoma rutila (Passeriformes: Cotingidae) en el desierto del Monte central, Argentina
}

\section{Diet of Phytotoma rutila (Passeriformes: Cotingidae) in the central Monte desert, Argentina}

\author{
Juan Manuel Rios ${ }^{1,2 *}$, Agustín Zarco ${ }^{3}$, María E. Mosca-Torres ${ }^{3} \&$ Pablo Sabat $^{1}$ \\ ${ }^{1}$ Departamento de Ciencias Ecológicas, Facultad de Ciencias, Universidad de Chile, Casilla 653, Santiago, Chile. \\ ${ }^{2}$ Instituto Argentino de Nivología, Glaciología y Ciencias Ambientales (IANIGLA), CCT Mendoza-CONICET, Z.C. 330 (5500) \\ Mendoza, Argentina. \\ ${ }^{3}$ Instituto Argentino de Investigaciones de Zonas Áridas (IADIZA), CCT Mendoza-CONICET, CC 507, 5500 Mendoza, \\ Argentina. \\ *E-mail: jmriosrama@gmail.com
}

\begin{abstract}
RESUMEN
Se determinó la composición botánica de la dieta del Cortarramas Phytotoma rutila en el desierto del Monte central, Argentina, mediante análisis microhistológico de las heces. En ambos sexos, la dieta estuvo compuesta por una importante proporción de frutos (26.8\%) y hojas (35.6\%) del arbusto Lycium tenuispinosum (Solanaceae) y hojas del árbol Prosopis flexuosa (Fabaceae) (26.8\%). P. rutila es una ave predominantemente folívora con un alto consumo de frutos durante el verano, cuando estos ítems son abundantes, lo que refleja su comportamiento oportunista.
\end{abstract}

Palabras Claves: Phytotoma rutila, dieta, Lycium tenuispinosum, hojas, frutos.

\begin{abstract}
Botanical composition of the diet of White-tipped Plantcutter Phytotoma rutila in the central Monte desert from Argentina, was determined by microhistological analysis of excreta. In both sexes, the diet was composed by a high proportion of fruits (26.8\%) and leaves (35.6\%) from the shrub Lycium tenuispinosum (Solanaceae) and leaves from the tree Prosopis flexuosa (Fabaceae) (26.8\%). P. rutila is a predominantly folivorous bird with a high consumption of fruit during summer, when these items are abundant, which shows an opportunistic behavior.
\end{abstract}

KeYwords: Phytotoma rutila, diet, Lycium tenuispinosum, leaves, fruits.

El Cortarramas (Phytotoma rutila, Vieillot 1818; Cotingidae) es posiblemente el ave herbívora de menor tamaño existente y su dieta incluye hojas, frutos y brotes (Bucher et al. 2003, De la Peña \& Pensiero 2003, De la Peña 2011). Al igual que sus congéneres $P$. rara y $P$. raimondii, que también son exclusivamente sudamericanos, $P$. rutila posee un pico aserrado que le permite 'mascar' las hojas y romper el parénquima foliar, haciendo más efectiva su digestión (López-Calleja \& Bozinovic 1999). Aunque una dieta rica en hojas es energéticamente pobre para aves paseriformes que no poseen una capacidad de fermentación significativa, estudios efectuados en $P$. rara sugieren que este género podría poseer una alta eficiencia digestiva facilitada por una elevada actividad enzimática intestinal lo que le permitiría procesar efectivamente alimentos nutricionalmente pobres (Meynard et al. 1999).
A pesar de que $P$. rutila está ampliamente distribuida en el sur de Sudamérica (Snow 2004) existen escasos estudios sobre sus hábitos alimentarios y ninguno de ellos realizado en zonas desérticas. Estudios previos sobre historia natural del ensamble de aves en la región fitogeográfica del desierto del Monte central argentino, proporcionan información valiosa sobre algunos aspectos de la ecología alimentaria y uso de hábitat de $P$. rutila en este ambiente. En un estudio acerca de la estructura gremial de las aves paseriformes, Lopez de Casenave et al. (2008) establecieron que esta especie pertenece a un gremio monoespecífico herbívoro-arborícola, recolectando materiales vegetales de árboles y arbustos. Otros estudios en este ambiente, determinaron que $P$. rutila se comporta como un migrador de verano: arriba a la porción central del desierto del Monte a principios de primavera, permaneciendo hasta el final del verano en densidades 
relativamente bajas del orden de 0,2 individuos por hectárea (Cueto et al. 2008). Estudiar la composición de la dieta de esta ave en el desierto del Monte es la primera aproximación para entender mediante qué mecanismos un ave migradora con una dieta herbívora, subsiste en un ambiente tan desfavorable cómo un desierto. Con el objetivo de estudiar los hábitos alimentarios de $P$. rutila, determinamos la composición botánica de la dieta natural analizando las excretas recolectadas de individuos de esta especie, cuantificando e identificando los ítems alimenticios consumidos.

El estudio se llevó a cabo en un bosque de Prosopis flexuosa dentro la Reserva de Biósfera de Ñacuñán $\left(34^{\circ} 02^{\prime}\right.$ S $67^{\circ} 58^{\prime}$ O), Mendoza, Argentina. El estrato arbóreo está conformado mayormente por Prosopis flexuosa y Geoffroea decorticans, mientras que el estrato arbustivo está constituido por Larrea divaricata, Lycium tenuispinosum, Capparis atamisquea, Condalia microphylla, Verbena aspera y Atriplex lampa. El estrato inferior está constituido por poáceas y dicotiledóneas herbáceas. El clima es seco y templado, con una precipitación media anual de $266 \mathrm{~mm}$, concentrada en primavera-verano (Ríos et al. 2012). Entre los años 2010 y 2011 se capturaron un total de 16 individuos adultos de $P$. rutila (hembras $\mathrm{n}=9$; machos $\mathrm{n}=7$ ) usando redes de niebla (ECOTONE). Luego de ser capturado, cada individuo fue colocado dentro de una bolsa de tela oscura y mantenido durante un período de tres horas en la sombra. Las excretas fueron recolectadas de la bolsa con pinzas y se vertieron dentro de viales plásticos conteniendo etanol $70 \%$ para su posterior análisis microhistológico en el laboratorio. Los individuos fueron pesados y liberados en el mismo sitio donde fueron capturados, salvo un individuo que fue sacrificado para estudiar el tracto digestivo que posteriormente fue depositado en la colección de aves del IADIZA. Las capturas se llevaron a cabo con permiso de la Dirección de Recursos Naturales Renovables, Gobierno de Mendoza.

Para determinar la dieta de $P$. rutila se aplicó la técnica microhistológica de Dacar \& Giannoni (2001). Se realizó un preparado por muestra de excreta de cada individuo, examinando sistemáticamente 80 campos microscópicos bajo un aumento de 400X. Para la identificación de los ítems alimenticios se utilizaron los preparados de referencia de hojas y frutos de las especies vegetales presentes en el sitio de estudio. Se calculó el porcentaje de ocurrencia de cada ítem consumido por cada individuo, diferenciando por sexo. Por último, se estimaron la media y el error estándar para todas las muestras analizadas con y sin discriminación de sexos. En todos los casos, los datos de composición de la dieta no se ajustaron a una distribución normal, por lo tanto para evaluar diferencias en las proporciones de especies vegetales consumidas, se usó la prueba no paramétrica de Kruskal-Wallis seguida por prueba de Dunn para comparaciones múltiples (Zar 1996). Para comparar las proporciones consumidas de hojas versus frutos por las hembras y por los machos, se utilizó la prueba pareada de Wilcoxon. Valores de $p<0.05$ fueron considerados significativos. Los promedios se muestran $\pm 1 \mathrm{EE}$.

Tanto hembras como machos, consumieron una mayor proporción de hojas que frutos $(Z=2.191, p=0.028$ y $Z=$ $2.366, p=0.017$; respectivamente. Fig. 1). De la fracción frugívora, los frutos de Lycium tenuispinosum (Solanaceae) resultaron ser el único ítem botánico en esta categoría, siendo consumidos en un $28.8 \%$ por las hembras y $24.8 \%$ por los machos (Tabla 1). Las hojas de L. tenuispinosum y de $P$. flexuosa también fueron ítems importantes en la conformación de la dieta de hembras y machos (Tabla 1). Los individuos hembras consumieron los ítems vegetales de manera diferente $(\mathrm{H}=48.46, p=0.040)$, siendo las hojas de L. tenuispinosum más consumidas que sus frutos y que las hojas de P. flexuosa. En cambio, para los machos no hubo diferencias entre los ítems más consumidos $(\mathrm{H}=35.31, p=$ 0.064). Al comparar el pool de individuos sin discriminar por sexo, hubieron diferencias en el consumo $(\mathrm{H}=63.458$, $p<0.001)$ siendo las hojas L. tenuispinosum el ítem más consumido (Tabla 1).

Aun cuando en este estudio no se determinó la selección de ítems tróficos por parte de esta especie, lo que requiere establecer la disponibilidad del alimento en el ambiente (Ríos et al. 2012), el consumo de frutos pareciera ser considerable. En este sentido, la alta proporción de frutos consumidos por $P$. rutila en este estudio puede explicarse teniendo en cuenta su morfología digestiva. Al igual que lo reportado en $P$. rara (Lopez-Calleja \& Bozinovic 1999), la morfología del tracto digestivo del individuo de $P$. rutila sacrificado, constituye el típico diseño-frugívoro: un estómago pequeño y el tracto intestinal corto y grueso. Por otro lado, consistentemente con lo reportado en otros paseriformes del Monte central (Ríos et al. 2012) la calidad nutricional del alimento pareciera tener un papel en la conformación de dieta de $P$. rutila en este ambiente árido. Los frutos de L. tenuispinosum contienen niveles altos de proteínas $(23,2 \%)$, azúcares $(27,1 \%)$ y agua (65\%, Orofino 2006). Estudios previos han reportado que la alimentación de $P$. rutila está asociada con el alto nivel de proteínas ( $29 \%$ ) contenidas en las hojas de Lycium cestroides en una región húmeda Chaqueña (Bucher et al. 2003) donde esta especie consume considerablemente menos frutos (7\%). En un estudio más reciente en la región Chaqueña, De la Peña (2011) determinó que $P$. rutila consume las hojas de L. cestroides triturándolas, mientras que a los frutos los consume enteros. De manera similar a lo encontrado en este estudio, los frutos de otra solanácea (Grabowskia boerhaaviifolia) resultaron tener importancia para la alimentación de $P$. raimondii en una región árida de Perú (Rosina \& Romo 2012). Aunque P. rutila una ave predominantemente folívora, en el desierto del Monte 
central muestra un alto consumo de frutos durante el verano, cuando estos ítems son abundantes, lo que refleja su comportamiento alimentario oportunista. Es posible que ante situaciones hídricas desfavorables, tales como las que ofrece un desierto, estas aves folívoras aumenten el consumo de alimentos con niveles altos en agua como los frutos y hojas ricas en agua preformada y metabólica. Sin embargo, son necesarios estudios adicionales sobre los mecanismos que subyacen a estos patrones de comportamiento alimentario para poder validar esta hipótesis.

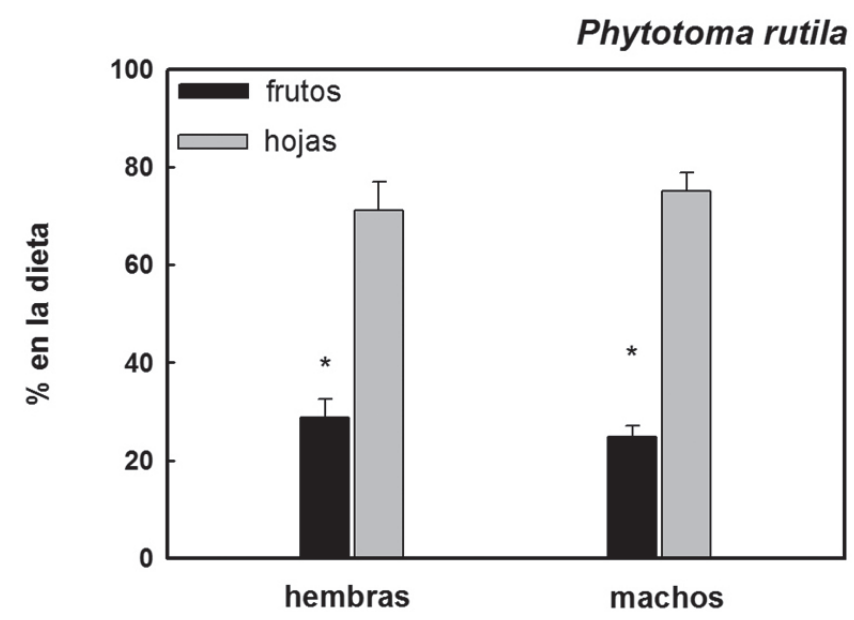

Figura 1. Conformación de la dieta de P. rutila en el desierto del Monte central. Los ítems dietarios están agrupados en categorías (frutos u hojas) consumidas según sexo. Los asteriscos indican diferencias significativas entre las categorías para cada sexo $(* p<0.05$; prueba de Wilcoxon).

Figure 1. Diet conformation of P. rutila in the central Monte desert. Dietary items are grouped into categories (fruits or leaves) consumed according to sex. Asterisks indicate significant differences between categories for each sex $(* p<0.05$; Wilcoxon test).

Tabla 1. Composición botánica de la dieta de Phytotoma rutila en el desierto del Monte central. Se muestra la composición dietaria (porcentaje promedio $\pm \mathrm{EE}$ ) para individuos adultos hembras, individuos adultos machos y para ambos sexos (porcentaje promedio).

TABLE 1. Botanical composition of the diet of Phytotoma rutila in the central Monte desert. Dietary composition (mean percentage \pm SE) for adult females and adult males, and also for both sexes (average percentage) is showed.

\begin{tabular}{|c|c|c|c|}
\hline \multirow[b]{2}{*}{ Especies } & \multirow{2}{*}{$\begin{array}{l}\text { Hembras }(\mathrm{n}=9) \\
\text { Proporción en la } \\
\text { dieta }(\%)\end{array}$} & \multirow{2}{*}{$\begin{array}{c}\text { Machos }(\mathrm{n}=7) \\
\text { Proporción en la } \\
\text { dieta }(\%)\end{array}$} & \multirow{2}{*}{$\begin{array}{c}\text { Ambos sexos }(\mathrm{n}=16) \\
\text { Proporción en la } \\
\text { dieta }(\%)\end{array}$} \\
\hline & & & \\
\hline \multicolumn{4}{|l|}{ Frutos } \\
\hline Lycium tenuispinosum & $28,8( \pm 3,7)^{\mathrm{A}}$ & $24,8( \pm 2,3)^{\mathrm{A}}$ & $26,8^{\mathrm{A}}$ \\
\hline \multicolumn{4}{|l|}{ Hojas } \\
\hline Lycium tenuispinosum & $34,3( \pm 2,4)^{\mathrm{B}}$ & $36,8( \pm 3,8)^{\mathrm{A}}$ & $35,6^{\mathrm{B}}$ \\
\hline Prosopis flexuosa & $24,9( \pm 3,2)^{\mathrm{A}}$ & $28,7( \pm 4,1)^{\mathrm{A}}$ & $26,8^{\mathrm{A}}$ \\
\hline Capparis atamisquea & $9( \pm 3,1)$ & $3,5( \pm 1,9)$ & 4,2 \\
\hline Solanum sp. & $2,7( \pm 1,1)$ & $5,7( \pm 1,2)$ & 6,3 \\
\hline Verbena aspera & $0,13( \pm 0,08)$ & 0 & 0,06 \\
\hline Chenopodium papulosum & 0 & $0,34( \pm 0,18)$ & 0,17 \\
\hline
\end{tabular}

Diferentes letras mayúsculas muestran diferencias significativas entre las proporciones de especies vegetales consumidas por las aves (prueba múltiple de Dunn). Sólo fueron analizadas y comparadas estadísticamente las especies vegetales que fueron consumidas por encima del $10 \%$. 


\section{AGRADECIMIENTOS}

Este trabajo fue financiado por FONDECYT N³130429 (JM Ríos). Agradecemos a Paola Sassi y Marisa Rosi por ayudar en la identificación botánica y a Victor Cueto por su apoyo.

\section{BIBLIOGRAFÍA}

Bucher, E.H., Tamburini D., Abril A. \& Torres P. 2003. Folivory in the White-tipped Plantcutter Phytotoma rutila: seasonal variations in diet composition and quality. Journal of Avian Biology 34:211-216.

Cueto, V.R., Lopez de Casenave, J. \& Marone, L. 2008. Neotropical austral migrant landbirds: population trends and habitat use in the central Monte Desert, Argentina. The Condor 110:70-79.

DACAR, M.A. \& GiANNONI, M. 2001. A simple method for preparing references slides of seed. Journal of Range Management 54:191-193.

DE LA PEÑA, M.R. 2011. Observaciones de campo en la alimentación de las aves. En: Biológica. Naturaleza, Conservación \& Sociedad. Edición especial No 13, p 88. Museo Provincial de Ciencias Naturales Florentino Ameghino, Argentina.

De la Peña, M.R. \& Pensiero, J.F. 2003. Contribución de la flora en los hábitos alimentarios de las aves en un bosque del centro de la provincia de Santa Fe, Argentina. Ornitología Neotropical 14:499-513.

SNow, D.W. 2004. Family Cotingidae (Cotingas). En: Handbook of the Birds of the World. (Eds. Del Hoyo, J., Elliot A. \& D. Christie), pp 32-66. Lynx Editions, Barcelona.

Lopez de Casenave, J., Cueto, V.R. \& Marone, L. 2008. Seasonal dynamics of guilds in a bird assemblage of the central Monte desert. Basic and Applied Ecology 9:78-90

Lopez-Calleja, M.V. \& Bozinovic, F. 1999. Feeding behavior and assimilation efficiency of the Rufous-tailed Plantcutter: a small herbivore. The Condor 101:705-710.

Meynard, C.M, Lopez-Calleja, M.V. \& Bozinovic, F. 1999. Digestive enzymes of a small avian herbivore, the Rufoustailed Plantcutter. The Condor 101:904-907.

Orofino, A.G. 2006. Preferencia alimentaria de dos especies de roedores del desierto del Monte: Estudios conductuales en laboratorio. Tesis doctoral. Universidad Nacional de Cuyo, Mendoza, Argentina.

Ríos, J.M., Mangione, A.M. \& Marone, L. 2012. Effects of nutritional and anti-nutritional properties of seeds on the feeding ecology of seed-eating birds of the Monte desert, Argentina. The Condor 114:44-55.

Rosina, M. \& Romo, M. 2012. Reproducción y alimentación de Phytotoma raimondii, cortarrama peruana en El Gramadal, Ancash. Revista Peruana de Biología 19:167-173.

ZAR, J.H. 1996. Biostatistical analysis (3rd ed.). Upper Saddle River: Prentice Hall.

Recibido: 26.07 .13

Aceptado: 19.12.13 